2002s-55

\title{
Experimenting with Gnutella Communities
}

\author{
Jean Vaucher, Gilbert Babin, Peter Kropf, \\ Thierry Jouve
}

Série Scientifique

Scientific Series

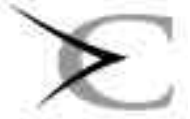

CIRANO

Montréal

Mai 2002 


\section{CIRANO}

Le CIRANO est un organisme sans but lucratif constitué en vertu de la Loi des compagnies du Québec. Le financement de son infrastructure et de ses activités de recherche provient des cotisations de ses organisationsmembres, d'une subvention d'infrastructure du ministère de la Recherche, de la Science et de la Technologie, de même que des subventions et mandats obtenus par ses équipes de recherche.

CIRANO is a private non-profit organization incorporated under the Québec Companies Act. Its infrastructure and research activities are funded through fees paid by member organizations, an infrastructure grant from the Ministère de la Recherche, de la Science et de la Technologie, and grants and research mandates obtained by its research teams.

\section{Les organisations-partenaires / The Partner Organizations}

-École des Hautes Études Commerciales

-École Polytechnique de Montréal

-Université Concordia

-Université de Montréal

-Université du Québec à Montréal

-Université Laval

-Université McGill

-Ministère des Finances du Québec

-MRST

-Alcan inc.

- AXA Canada

-Banque du Canada

-Banque Laurentienne du Canada

-Banque Nationale du Canada

- Banque Royale du Canada

- Bell Canada

- Bombardier

- Bourse de Montréal

-Développement des ressources humaines Canada (DRHC)

-Fédération des caisses Desjardins du Québec

-Hydro-Québec

-Industrie Canada

-Pratt \& Whitney Canada Inc.

-Raymond Chabot Grant Thornton

-Ville de Montréal

(C) 2002 Jean Vaucher, Gilbert Babin, Peter Kropf et Thierry Jouve. Tous droits réservés. All rights reserved. Reproduction partielle permise avec citation du document source, incluant la notice $@$.

Short sections may be quoted without explicit permission, if full credit, including (C) notice, is given to the source.

Les cahiers de la série scientifique (CS) visent à rendre accessibles des résultats de recherche effectuée au CIRANO afin de susciter échanges et commentaires. Ces cahiers sont écrits dans le style des publications scientifiques. Les idées et les opinions émises sont sous l'unique responsabilité des auteurs et ne représentent pas nécessairement les positions du CIRANO ou de ses partenaires.

This paper presents research carried out at CIRANO and aims at encouraging discussion and comment.

The observations and viewpoints expressed are the sole responsibility of the authors. They do not necessarily represent positions of CIRANO or its partners.

\section{ISSN 1198-8177}




\title{
Experimenting with Gnutella Communities
}

\author{
Jean Vaucher ${ }^{*}$, Gilbert Babin ${ }^{\dagger}$, Peter Kropf ${ }^{*}$, and Thierry Jouve ${ }^{\S}$
}

\section{Résumé / Abstract}

Les réseaux informatique ainsi que les systèmes distribués peuvent être considérés comme des communautés où les composantes - que ce soit des systèmes complets, des programmes ou des usagers interagissent dans un environnement partagé. Ces communautés sont dynamiques car des éléments peuvent s'y joindre ou quitter en tout temps. L'article présente les résultats d'une suite d'expériences et de mesures faites sur Gnutella, un système peer-to-peer à grande échelle qui opère sans aucun contrôle centralisé. Nous avons remarqué qu'une grande partie des messages échangés sont erronés ou redondants et que les interactions entre nœuds ne durent pas très longtemps. En particulier, des connexions durant plus d'une minute sont des phénomènes rares. Les nœuds passent donc la majorité de leur temps à remplacer les partenaires perdus et, contrairement à l'idée répandue que les réseaux peer-to-peer sont immenses, nous avons noté que les communautés effectives étaient assez limitées. Gnutella est un environnement très dynamique avec peu de stabilité. Par exemple, de 42,000 sites avec lesquels nous avons établi une connexion, il a seulement été possible de re-communiquer de façon régulière avec 57. Dans un tel environnement, la chance joue un rôle important dans la performance observée; mais nous avons élaboré un protocole expérimental permettant de comparer diverses options.

Computer networks and distributed systems in general may be regarded as communities where the individual components, be they entire systems, application software or users, interact in a shared environment. Such communities dynamically evolve with components or nodes joning and leaving the system. Their own individual activities affect the community's behaviour and vice-versa. This paper discusses various experiments undertaken to investigate the behaviour of a real system, the Gnutella network, which represents such a community. Gnutella is a distributed Peer-to-Peer data-sharing system without any central control. It turns out that most interactions between nodes do not last long and much of their activity is devoted to finding appropriate partners in the network. Good connections lasting longer appear only as rare events. For example, out of 42,000 connections only 57 hosts were found to available on a regular basis. This means that, in contrast to the common belief that this kind of peer-topeer networks or sub-communities are always large, they are actually quite small. However, those subcommunities examplify very dynamic behaviour because their actual composition can change very quickly. The experimental results presented have been obtained from a Java implementation of Gnutella running in the open Internet environment, and thus in unknown and quickly changing network structures heavily dependent on chance.

Mots-clés : Gnutella, réseaux peer-to-peer, communautés virtuelles, internet, systèmes distribués, protocoles de télécommunication

Keywords: Gnutella, peer-to-peer networks, Internet communities, distributed systems, protocols

\footnotetext{
* Département d'Informatique et recherche opérationnelle (DIRO), Université de Montréal, Montréal, Canada et CIRANO, Centre interuniversitaire de recherche en analyse des organisations

† CIRANO et HEC, Montréal

*IRANO et DIRO, Université de Montréal, Montréal, Canada

${ }^{\S}$ DIRO, Université de Montréal, Montréal, Canada
} 


\title{
Experimenting with Gnutella Communities *
}

\author{
Jean Vaucher ${ }^{1}$, Gilbert Babin ${ }^{2}$, Peter $\operatorname{Kropf}^{1}$, and Thierry Jouve ${ }^{1}$ \\ 1 Informatique et recherche opérationnelle \\ Université de Montréal, Montréal, Québec, Canada \\ \{vaucher,kropf,jouvethi\}@iro.umontreal.ca \\ 2 Technologies de l'information \\ HEC - Montréal, Montréal, Québec, Canada \\ Gilbert.Babin@hec.ca
}

\begin{abstract}
Computer networks or distributed systems in general may be regarded as communities where the individual components, be they entire systems, application software or users, interact in a shared environment. Such communities dynamically evolve with components or nodes joining and leaving the system. Their own individual activities affect the community's behavior and vice versa. This paper discusses various practical experiments undertaken to investigate the behavior of a real system, the Gnutella network, which represents such a community. Gnutella is a distributed Peer-to-Peer data-sharing system without any central control. It turns out that most interactions between nodes do not last long and much of their activity is devoted to finding appropriate partners in the network. Good connections lasting longer appear only as rare events. For example, out of 42,000 connections only 57 were found to be up over a certain time period. This means that in contrast to the common believe that this kind of peer-to-peer networks or sub-communities are always very large, they are actually quite small. However, those sub-communities expose a very dynamic behavior because their actual composition can change very quickly. The experimental results presented have been obtained from a Java implementation of Gnutella running in the open Internet environment, and thus in unknown and quickly changing network structures heavily depending on chance.
\end{abstract}

\section{Introduction}

Whenever a set of autonomous individuals acts in a shared environment, interaction emerges which may result in manifold relations between the individuals and/or groups of individuals. Those relations and the associated behavior of individuals and groups may induce structures to the groups. Such structures are commonly called communities. The behavior of biological individuals, such as ants or bees, but also humans has been widely studied in the social sciences [4]. Key findings include that despite the largely varying (intellectual) capacities of individuals and groups, a set of common characteristics for acting in a shared environment still may be observed [9]. However,

\footnotetext{
* A shorter version of this paper is to appear in the Proceedings of the 4th Conference on Distributed Communities on the Web (DCW 2002), Lecture Notes in Computer Science series, Springer-Verlag.
} 
this usually depends on the specific knowledge of the individuals and their time already spent within a community. Among the characteristics identified, we find that,

- each individual can identify a few members of a community and may exchange information with them;

- there exists no single individual that knows or controls the whole community;

- some individuals may be more "intelligent" than others and have more and/or better information;

- communities are often hierarchically structured with one or more leading individuals.

Communities continuously evolve in some evolutionary process starting with just a few individuals (at least two). The resulting set of inter-related community members is generally called the social network of a community. While the number of individuals in a community can grow very fast, the single individual needs only little information about other individuals to still be able to potentially interact with a large number (or all) of the community members. The six degrees of separation property [14] illustrates this in the case of human communities. Moreover, communities are often characterized by a highly self-organizing behavior. Insect collectives such as ants or bees, but also physical and chemical systems composed of large numbers of individuals or particles interact locally and contribute thereby to global organization, optimization and adaptation to the environment.

Computer networks or distributed systems in general may be regarded as communities similar to the above examples. Most obviously, the Internet or Web forms entities that can be characterized as communities. Many approaches to define communities on the Web $[6,8,10]$ are based on the use of existing link patterns and they therefore lack the characteristic community properties to adapt to the current context and to dynamically evolve. Implicit information $[9,13]$ other than link patterns are necessary to achieve this.

A number of applications have been developed which include in one way or another the idea of communities on the Internet. Among those are Yenta [7], an agent based system to find people with similar interests and to make them known to each other, Freenet [5], an information publication system storing, caching and distributing information on demand without any centralized control, or Gnutella [2], a distributed Peer-to-Peer data-sharing system. In the Gnutella system, a user only needs to know one (or several) other participants to join the community. The mechanism to propagate information (search for partners or particular data) refrains from any central control: it is based on a passing on mechanism from participant to participant. The thereby formed community is highly dynamic as participants can join and leave at any time without having to contact any administrative unit. The very simple Gnutella protocol [1] provides the rules for the exchange of information.

In order to investigate the behavior of communities on the Internet, the Gnutella system has been chosen for the research presented in this paper. The system provides an ideal practical testbed because all the participating individuals are unknown, no central control exists, and the community is sufficiently large. In fact, the only common component is the communication protocol and the core system behavior where each 
participant acts as a client and a server at the same time while applying the aforementioned information propagation mechanism.

The next two sections introduce the Gnutella system and protocol. In section 4 we describe the Jtella platform used for the experiments which are presented in detail in section 5. The method applied for measuring the performance of Gnutella applications and the results observed are discussed in section 6 before concluding with a discussion about the various experimental results observed.

\section{Overview of Gnutella}

Gnutella is a distributed Peer-to-Peer (P2P) application for the sharing of files over the Internet. It was designed as a replacement for Napster [22] and has been used mainly for the dissemination of multimedia files.

Each participant in a Gnutella network runs a program on his computer that acts both as a client and a server as well as a router. Gnutella programs are referred to as servents (SERVer+cliENT), nodes or simply clients. As a client, the application provides an interface where a user can enter keywords describing the files that he is seeking. The program then sends the request to neighbouring participants who pass it on to their neighbours who do the same; thus propagating it throughout the network. At the same time, clients check to see if the request corresponds to local files they are willing to share and, if so, they send back a response. File transfers are done via another route using standard HTTP protocol requests.

The fundamental feature of Gnutella is that it does not rely on centralized databases or proprietary software. It also tries to ensure a measure of anonymity. As a result, it is resistant to both hardware failure and legal attack. The first Gnutella application was released in March 2000 but it was officially available for only a 24 hour period [2]. The basic protocol is quite simple; it was extracted from the original software and is now available on the Web [1]. Although, it has been reported to suffer from performance and scalability problems [18], the Gnutella protocol has resulted in a large number of implementations.

Initially, as a replacement for Napster, the Gnutella network grew exponentially. This growth has been charted by several researchers [3,17]. Available data shows the network growing from around 1,000 nodes in November 2000 to over 40,000 in June 2001. Over this period, Ripeanu [17] found that over 400,000 different users had connected to Gnutella. In another study, Saroiu's crawler found over 1 million different host addresses in an 8 day period [21]. However, since the summer of 2001, the network has been steadily shrinking, reaching an average of 16,000 users in January 2002 [3]. One can surmise that, if the main interest in Gnutella was sharing of music, many users have switched to more efficient specialised services such as Morpheus-KaZaA from MusicCity which now claims to have over 300,000 simultaneous users [15].

As the first widespread decentralised protocol, Gnutella is worthy of study. Its open nature and simple basic protocol also make it easy to use in experiments. Because the protocol is not specifically oriented to a single application domain (like mp3encoded music), it is also easy to use Gnutella as a low level dissemination or broadcast protocol upon which to piggy-back other applications - with specially formatted 
query/response strings. Parallel private Gnutella networks can also be set-up by the simple expedient of using private bootstrap host caches.

\section{Description of the Gnutella Protocol}

Each participant in a Gnutella network maintains a small number of permanent links to neighbours (typically 4 or 5). Search is done via flooding - a distributed form of broadcast. Messages are sent to the neighbours who pass them on to their neighbours and so on. The number of hosts which are contacted in this way increases exponentially with each jump. In order to limit the potential data explosion, the number of jumps is bound by a time-to-live (TTL) counter which is decremented on each passing on. When the counter reaches zero, the message is no longer propagated. Messages also have a hop counter to keep track of how far they have come.

Gnutella provides also for some notion of anonymity. Specifically, queries do not contain the identity of the initiating host. Instead, each Gnutella message has a unique identifier (ID) and propagating hosts maintain routing tables keyed on this ID which indicate from which connection a message arrived. Answers carry the same ID and are returned along the same route as the query. The anonymity is only relative because downloads are done directly without passing through the Gnutella connections. The routing tables are also useful in preventing looping and duplicating messages: if the ID of a query (not an answer) is already present in the table, the message is seen to be a duplicate and is not propagated.

The Gnutella protocol is based on four types of messages (actually there is a $5^{\text {th }}$ type to deal with firewalls, but it is not pertinent to our discussion). The messages come in pairs: one for the requests and one for the answers. One pair is used to search for files; the other to obtain the addresses of active participants.

The file search messages are:

Query - contains the user request as an unformatted string of keywords ${ }^{1}$;

Reply - is used by a host to return a list of matching files along with a short description of each file as well as the Host:Port address to be used for an HTTP download.

The next two messages are used to discover the addresses of participating hosts:

Ping - a request for host addresses;

Pong - a reply to the Pong with a Host:Port address along with extra information about the host bandwidth and the number of local files.

According to the protocol, a host receiving a Ping should answer with its own address in a Pong as well as forwarding the Ping to its neighbours. In practice, to reduce overhead, a host which already has too many neighbours, may pass on the message without returning its own address. Some hosts may act as central directories. They do not propagate Pings; rather, they maintain a cache of addresses they have received and

\footnotetext{
${ }^{1}$ Note: in possible extensions of Gnutella to specialized areas, one would expect the format and semantics of the Query request to be more tightly defined.
} 
return a small number of these. There is a number of sites well-known to the Gnutella community which act as directories. This is how initial connection to Gnutella usually operates. However, any active host can serve as an initial connection point.

Finally, the protocol gives details about the handshake to be used on initial connection and suggests that Gnutella applications use port 6346 as the server address.

It is interesting to note that the protocol only specifies the minimum necessary to maintain connectivity, broadcast queries and receive replies. The other aspects of the functionality that we expect in any program that accesses the Gnutella network either rely on other protocols or are left unspecified. File transfers, for example, are done out of band - using the established HTTP GET protocol; furthermore, details of how to index files and match queries to local files are also left undefined.

\section{The Experimental Platform}

Our experimental platform is based on Jtella, an Gnutella framework written in Java by Ken McCrary $[11,12]$. In the author's words:

The Jtella API project is an effort to create a simple, easy to use Java programming interface for the GNUTella network. The goal is to make it easy to produce Java applications and tools for accessing the GNUTella network.

Jtella is made up of about 40 classes and 7000 lines of Java. It manages the initial connection to the Gnutella network, the maintenance of a specified number of connections and the routing of messages. An indication of the ease of use is that simple applications to search or monitor traffic require less than 150 lines of Java (on top of the Jtella Framework). Note that Jtella does not cover the indexing of files, matching queries or media playing. Jtella served as our introduction to the implementation of the Gnutella protocol but we took the liberty of rewriting or modifying about 1000 lines mainly dealing with parallelism and synchronization. We also uncovered, reported [23] and bypassed a Java bug: threads which are not started are not garbage collected.

\subsection{Architecture of Jtella}

The main building blocks of Jtella are: the Connection objects, the Router, the Connection managers and the Host Cache. The architecture is shown in Fig. 1. It is quite similar to that of LimeWire [19].

There is one Connection object for each connection. Each Connection is a Thread that handles incoming messages and puts them on a common message queue for the Router. Each Connection also has a second Thread with a message queue to handle output messages.

The Router is a separate Thread. It takes messages off its queue, checks them against a table of recently seen messages and, if they are not duplicates, it places them in the appropriate output queues. The router tables are also used to return answers via the connection the query came from. For efficiency, in our version of Jtella, queues are fixed in length: the router queue has 200 slots and the connection queues are 50 elements long. In general their occupation is less than $5 \%$. 


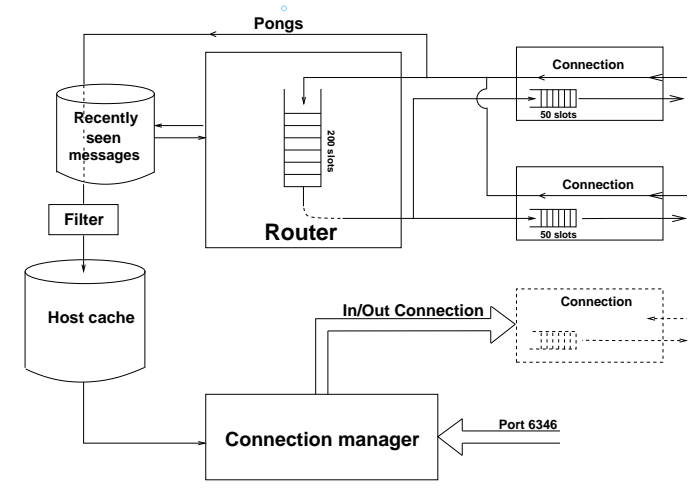

Fig. 1. Servent Architecture

There are two connection managers whose job it is to maintain a specified number of active connections. Initially, Jtella was set up to keep 4 connections open: 2 outgoing and 2 incoming. If an incoming connection failed, the following incoming connection would be accepted. If the number of outgoing connections was below the specified level, for every missing connection, the outgoing manager would launch 2 start-up threads to try to connect to new hosts. The current version is more flexible in the split between incoming and outgoing connections. By default, two slots are reserved: one incoming and one outgoing, but the others can be of either type. When a connection fails, we launch two connector threads and, at the same time, we accept incoming connections. If all connection attempts succeed, we can temporarily have too many connections; but connections die quickly and this excess capacity is short lived. On start-up, all connections are necessarily outgoing but in a short time, as our address is made known through Pongs, the rate of incoming requests increases to the point that most failed connections are replaced by incoming connections.

To discover addresses of Gnutella participants, we send out a Ping whenever we open a new connection, and we put the addresses from all Pongs received - not just replies to our Ping - into a cache. Because we receive many more host addresses than we can use, we limit our cache to 200 addresses and discard the others. As will be discussed later, most addresses that we receive are invalid. An important modification to Jtella was the addition of a filter to weed out bad addresses. When the cache is empty or low, we connect to well known host caches which use the same Ping/Pong messages as all other Gnutella nodes but whose sole function is to store addresses and redistribute them later to callers. Recently, this host cache function has been partially delegated to the network and in many servent implementation, whenever a node refuses a connection request, it sends back a number of Pongs from its host cache before shutting down the connection. Commonly, some servents send back 10 Pongs and others 50.

In our version of Jtella, the system keeps the following statistics on all connections for each run:

- connection type (IN or OUT), 
- time started,

- set-up time until failure or first message,

- duration of connection,

- number of messages received: pings, pongs, queries, and replies,

- termination code.

\section{Gnutella Measurements}

With Gnutella, performance for any one session is highly dependent on chance. If a client happens to find reliable hosts early, it will obtain a steady flow of messages. At other times, it may struggle to find even a single permanent connection and it is not rare for identical servants run in parallel to have 2:1 differences in performance indicators.

Before proceeding to more exact measurements and tests, we present typical output from two exploration experiments which show the difficulty in maintaining connectivity and the difficulty in quantifying behavior.

\subsection{Exploration Experiment I}

Our principal measurement program named TestServent sets up a passive node with a specified number of connections, routes messages and collects statistics. It also prints out the current status of the node every 15 seconds. Typical output is shown in Figure 2.

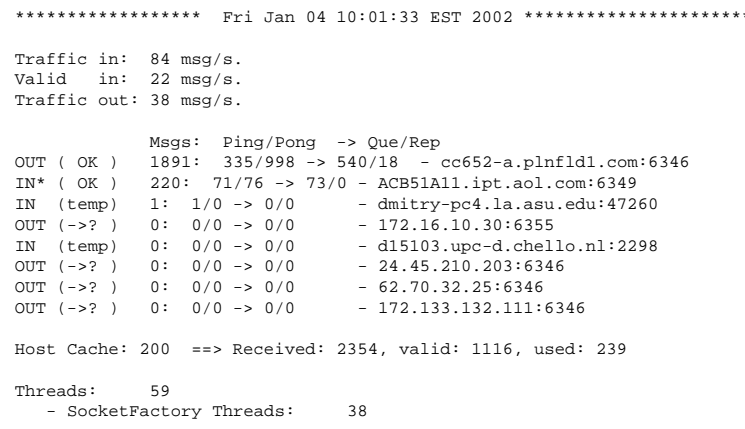

Fig. 2. Partial Output of Program TestServent

The first lines show the average traffic since the previous printout. The first thing to notice is that while 84 messages per second were received, most were invalid (either duplicates and Pongs with incorrect addresses); this left 22 valid messages which gave rise to 38 output messages.

Next, we see a snapshot of the connection activity. These are listed in the order in which they were created. OUT connections are created by our client, whereas IN connections were initiated by other hosts. For each connection, we give a status code (i.e., 
OK), list the number of messages received (total then categorized), and finally give the address of the corresponding host. In this test, we were trying to maintain 4 active connections, but at this moment there are 8 connections with only two in normal operation (OK). The other 6 connections are in various states of initialization or termination.

The first line shows the oldest connection, which has received 1891 messages and has been in operation for about 25 seconds. Only 18 messages are replies to queries. Typically, more messages are concerned with maintaining connectivity (Pings and Pongs) than with searching for information.

The second line shows an active input connection. The "**" indicates that the connected host has responded to our Ping with a Pong reporting its public port number (6349). Of the other 6 connections, two, noted temp, are incoming connections which we decided to refuse (probably because 4 connections were up when they first arrived). We are keeping those temporarily open while returning some host addresses and waiting for them to answer our Ping. The other 4 are output connections in the process of opening a socket (->?).

The next line shows the state of the host cache. At present, it is full (200 addresses). 2354 Pongs were received but of those, less than half (1114) had valid distinct addresses. This number is more than enough because only 239 were used to open new outgoing connections or passed on to other nodes.

The last line shows the parallelism involved in Gnutella and underlines a Java problem. With Java 1.3, a Thread trying to open a socket to an inaccessible host may be blocked for up to 13 minutes. Jtella creates SocketFactory Threads for this task and assumes they have failed if they don't succeed in 10 seconds. The printout shows that 38 threads are trying to open sockets, that is 34 blocked threads in addition to the 4 (-> ?) in the active list.

This brief look at Gnutella underlines a fundamental aspect of the network: most connections do not last long and much of a client's activity is dedicated to finding replacements. In later sections, we will study this aspect more closely.

\subsection{Exploration Experiment II}

Figures 3 and 4 illustrate the stochastic nature of Gnutella. We ran two Gnutella sessions in parallel for 45 minutes and monitored two parameters: the number of messages received per second and the horizon, a measure of network size. More precisely, every minute, we broadcast a Ping and then we tally all the answering Pongs over the next 60 seconds.

Figure 3 shows the input rate while Figure 4 shows the horizon for the two clients. Both clients attempt to keep 4 connections open. The graphs are quite noisy but it is clear that Client B has done better than Client A. The average message rate for B is around 180 compared to 120 for $\mathrm{A}$.

The random nature of operation is even more pronounced in the measurement of the horizon. It is hard to believe that these results were obtained from two identical programs run under identical conditions. This also shows the difficulty in trying to estimate the size of the Gnutella network.

In what follows, we present the results from experiments designed to quantify some critical aspects of Gnutella operation, namely: 


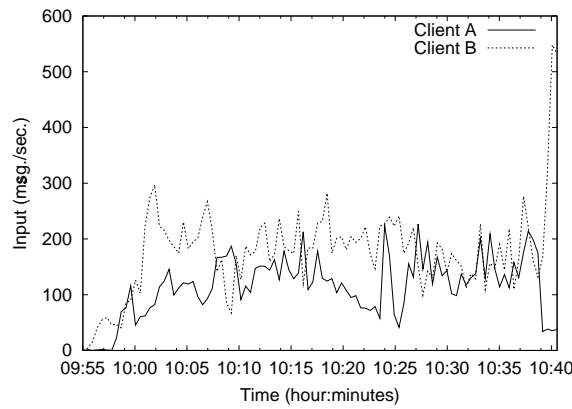

Fig. 3. Input Flow vs. Time for Two Clients

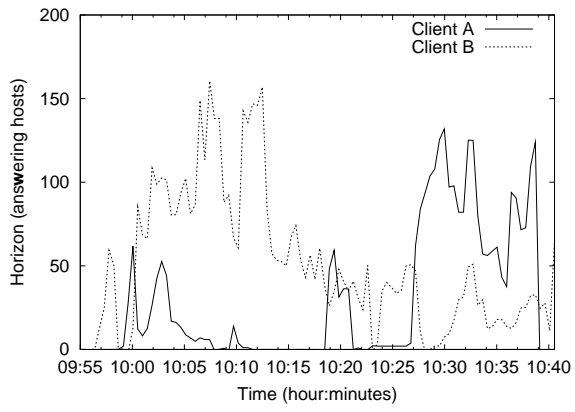

Fig. 4. Horizon vs. Time for Two Clients

- the validity of Pong information,

- the success rate in creating connections,

- the duration of connections,

- the proportions of various message types,

- the aging of addresses, and

- transmission delays.

In all cases, we present results from 2 or more experiments to give an idea of typical behavior as well as variability. Although it is difficult to obtain exact meaningful measures of performance, our results still lead to interesting conclusions.

Later on, in Section 6, we present an experimental protocol that we used to overcome the stochastic nature of Gnutella in order to show the effect of an operating parameter, the number of active connections, on performance.

\subsection{Validity of Pong Addresses}

Gnutella hosts depend on the Ping/Pong mechanism to discover the addresses of participating hosts. Unfortunately, early trials showed that many (if not most) addresses provided by Pongs are useless.

First, many addresses are duplicates. In experiments done around Oct. 16th, 2001, between $75 \%$ and $88 \%$ of addresses received were identical to addresses already present in our cache of 200 addresses. In one trial with 2390 Pongs received, a single address (32.101.202.89) was repeated 210 times. Fortunately, a simple test can be used to eliminate a large proportion of duplicates because about $25 \%$ of addresses are identical to the one received immediately before.

Secondly, many addresses are "special" values which are obviously invalid:

- 0.0.0.0: default network address,

- 127.0.0.1: loopback address,

- 255.255.255.255: broadcast address.

There are also blocks of Internet addresses which are reserved for particular uses and make no sense in the context of the Gnutella network. One example is multicast addresses but the most common problem results from hosts operating on private internets 
with NAT (Network Address Translation) translation [16]. These use addresses which have no global validity. The invalid formats we identified are the following:

$-224.0 .0 .0-240.255 .255 .255$ (multicast),

- $10.0 .0 .0-10.255 .255 .255$ (private addresses),

- $172.16 .0 .0-172.31 .255 .255$ (private addresses),

- $192.168 .0 .0-192.168 .255 .255$ (private addresses).

Table 1 shows the results from 2 experiments where we collected and analyzed all Pong addresses.

Table 1. Classification of IP Addresses Received in Pong Messages

\begin{tabular}{|c|c|c|c|}
\hline & \multicolumn{3}{|c|}{ Experiment $A$ Experiment $B$} \\
\hline$\overline{\text { Total addresses received }}$ & 7482 & & 19484 \\
\hline Invalid addresses & 2240 & $(30 \%)$ & $7042(36 \%)$ \\
\hline Repeated addresses & 1432 & $(19 \%)$ & $5298(27 \%)$ \\
\hline Already in cache & 1696 & $(23 \%)$ & $3978(20 \%)$ \\
\hline Retained & 2114 & $(28 \%)$ & $3166(16 \%)$ \\
\hline Unique good addresses & 1514 & $(20 \%)$ & $1792(9 \%)$ \\
\hline
\end{tabular}

As a result of these experiments, we modified the cache algorithm in our client to filter out invalid and repeated addresses as well as those already in the cache. With these mechanisms in place, the data above shows that between $16 \%$ and $28 \%$ of addresses would be retained. Due to the limited size of the cache, not all duplicates can be detected. The last line of the table - the results of off-line analysis of all addresses received - shows the actual proportion of unique valid addresses to vary between $9 \%$ and $20 \%$.

Even with this drastic filtering and the use of a small cache, we normally receive many more addresses than we need. The following extract of the output from a recent lengthy execution of TestServent shows that our early conclusions are still correct: only $30 \%$ of the addresses were valid and even then we received 4 times the number that we required .

Host Cache: 197 ==> Received: 59719, valid: 17489, used: 4145

\subsection{Creating Sockets}

Having filtered out invalid addresses, we then considered the probability of success in connecting to hosts whose addresses we retained. There are several reasons why a connection attempt could fail: the host may be too busy and refusing connections, the application may have terminated or the computer been disconnected from the network.

A problem that appeared early in our study of Gnutella - with programs written in Java - is that threads that tried to open sockets to unavailable hosts would often freeze. Further study showed that these threads were waiting for a timeout which in our set-up (Java 1.3 and Linux 2.2.17) could take up to 790 seconds (13 minutes). In 
Windows environments, a time-out of 45 seconds was reported. To minimize the effect of this blocking, a separate thread is used for socket creation and the main loop waits a maximum of 10 seconds before trying to connect to another address.

In one 90 minute session, our servent attempted to connect to 2541 hosts. Here is the breakdown of the results obtained and the average time to set-up the connection:

- $31 \%$ : success - connection achieved in $2.3 \mathrm{sec}$.,

- $20 \%$ : failure reported rapidly in $1.7 \mathrm{sec}$.,

- $49 \%$ : blocking, failure noted after $10 \mathrm{sec}$.

To study more closely this phenomenon, we created a Connection tester (CTester) that takes a list of host:port addresses and tries to open a socket to each - which it then closes without attempting to do a Gnutella handshake. For each connection, it prints out the time until the socket creation terminates as well as the error message if the socket could not be created. For this test, we used 100 random addresses taken from a TestServent $\log$ file. Here are the results:

- $36 \%$ : socket created in $1.6 \mathrm{sec}$. (9 sec. maximum),

- $26 \%$ : rapid failure in 0.9 sec.,

- $38 \%$ : blocking and failure reported after 790 sec.

The blocking during socket creation in Java explains the difficulty reported by several researchers who implemented crawlers to analyze the topology of Gnutella. Given the data above, where roughly, one connection attempt in three is blocked for $13 \mathrm{~min}$ utes, this means that a single thread can only examine about 4 addresses/minute and multi-threading is obviously a must.

\subsection{Duration of Connections}

We analyzed the log files from several sessions to determine how long connections stay valid once they have been established. In our longest test, maintaining 5 connections over 24 hours on Nov. 28th, 2001, 20,945 valid connections were observed. By valid, we mean that a socket connection was established and the handshake was successful. At the same time, 36,000 incoming requests were refused and 6,000 outgoing socket creations failed. The average duration for all sessions was $31 \mathrm{sec}$. and the average setup time was $0.21 \mathrm{sec}$. It is difficult to reason about an average connection, however, because the distribution is highly skewed and results are predicated by a small number of very large values. In this case, the longest session lasted about 11 hours $(39,973$ seconds) and 5 sessions lasted over 8 hours. Table 2 (Experiment C) gives an indication of the distribution of connection duration.

In a more recent experiment, maintaining 5 connections over 1 hour on Dec. 30th, 2001, there were 297 valid Gnutella sessions for which the average set-up time was $1.05 \mathrm{sec}$. and the average duration was 57 seconds. Again the distribution was highly skewed and results are tabulated in Table 2 (Experiment D).

The main conclusion is that the average duration of a connection is quite short, between 30 seconds and a minute. 
Table 2. Duration of Valid Connections

\begin{tabular}{lrr}
\hline & Experiment $C$ Experiment $D$ \\
\hline Average & $31 \mathrm{sec}$. & $57 \mathrm{sec}$. \\
Median & $0.17 \mathrm{sec}$. & $0.4 \mathrm{sec}$. \\
Std. dev. & $717 \mathrm{sec}$. & $319 \mathrm{sec}$. \\
Max. & $6350 \mathrm{sec}$. & $3233 \mathrm{sec}$. \\
\hline Average top 1\%: & $2973 \mathrm{sec}$. & $2960 \mathrm{sec}$. \\
Average top 10\%: & $307 \mathrm{sec}$. & $540 \mathrm{sec}$. \\
Average bottom $90 \%:$ & $0.26 \mathrm{sec}$. & $2.3 \mathrm{sec}$. \\
\hline
\end{tabular}

\subsection{Distribution of Message Types}

Table 3 shows the distribution of message types in 3 experiments. The first is the 24 hour session mentioned previously (Experiment C, Sect. 5.5). The other two (Experiments E and F) show the variability of the results.

Table 3. Message Type Distribution

\begin{tabular}{lrrr}
\hline & $\begin{array}{c}\text { Experiment C } \\
\text { (Nov. 28th, 2001) }\end{array}$ & $\begin{array}{c}\text { Experiment E } \\
\text { (Jan. 4th, 2002) }\end{array}$ & $\begin{array}{c}\text { Experiment } F \\
\text { (Dec. 18th, 2001) }\end{array}$ \\
\hline Messages received & $36,797,372$ & 278,871 & $5,965,273$ \\
\hline Pings & $9 \%$ & $11 \%$ & $9 \%$ \\
Pongs & $57 \%$ & $26 \%$ & $72 \%$ \\
Queries & $30 \%$ & $57 \%$ & $15 \%$ \\
Replies & $4 \%$ & $5 \%$ & $4 \%$ \\
\hline
\end{tabular}

The ratio of Pings and Replies is fairly constant (10\% and $5 \%$, respectively) but the proportion of Pongs and Queries varies widely. However, it is obvious that a major part of the Gnutella traffic is involved in overhead (Pings and Pongs) rather than search.

\section{7 “Good" Hosts}

Having determined that the majority of Gnutella participants are transients who only connect to the network occasionally and then for short periods, we then set forth to see if the reliable hosts that we identified during one session could be reused in future sessions. If so, one could dispense with the need to connect to the same well-known host caches on start-up.

First, we extracted "good" connections from experiments done over 24 hours on December 30th, 2001. Our criterion for selection of a "good" host address was one to which the connection had remained active for at least 2 minutes (over twice the average connection duration). From 41,789 recorded connections, 564 connections (1.3\%) were considered "good."

Next, we scheduled periodic executions of the CTester program to see if it was still possible to re-establish socket connections to the "good" hosts. Actually, there is a 
problem in trying to reuse old addresses from incoming connections because the socket port number that we obtain bears no relation to that host's public server port to which connection request should be directed. Generally, it will be 6346, the standard Gnutella port but not always. In the latest version of our client, we try to obtain the public port of all incoming connections by sending a Ping and waiting for a Pong with a hop count of 1 . Out of our 564 selected addresses, 191 (34\%) were incoming connections and of those only about a third (70) answered our Ping. In $75 \%$ of these cases the public port returned was 6346; justifying our choice of that address for hosts that do not answer. Parenthetically, the fact that two thirds of our "good" hosts never responded to a Ping shows the difficulty in trying to measure network size by Pinging hosts!

The day after the addresses were obtained, we scheduled experimental runs every four hours over a 24 hour period. After this, we ran the experiment once a day for a week. During the first two days, the success rate for reconnection dropped steadily from about $18 \%$ to $10 \%$. A week later, it reached $7 \%$ where it has remained - varying between $6.4 \%$ and $7.8 \%$.

This result may seem disappointing especially since in 380 cases $(67 \%)$ we were unable to reconnect even a single time. However, there were 4 hosts that we were always able to reach and another 57 who were available $50 \%$ of the time or better. Additional experiments showed that we could open Gnutella sessions to $90 \%$ of the hosts to which Ctester could open a socket. Thus it is possible to identify reliable semi-permanent Gnutella Hosts.

\subsection{Latency}

We also set up an experiment to measure the delays involved in the metering of network size. The results below were taken over 30 minutes on the afternoon (14:00) of Oct. 31, 2001.

Every minute, we created a separate thread which broadcast a Ping to all connections and collected all responding Pongs. We tabulated the delay and the hop count of the responses and we measured maximum, minimum and averages values.

As we have come to expect, there was wide variation between individual measurements. Once started, the number of responding hosts varied from a low of 5 to a high of 252. In the number of hops in the responding Pongs, we expected that the minimum would be 1 (from our neighbouring hosts); in fact, the minimum was often 2 and sometimes 3 even with over 50 responses. Obviously, some connected hosts choose not to respond to Pings. This is in keeping with one of the recommendations of a LimeWire technical paper [20] on a strategy to reduce the Ping/Pong overhead: only send out Pongs if you are willing and able to accept new connections.

The major result from the experiments was the delay in receiving responses. Response time varied from $0.9 \mathrm{sec}$. to a maximum of $128 \mathrm{sec}$. with a mean value of $13 \mathrm{sec}$. for a mean of 5 hops. Five hops means a total of 10 message transmissions (5 Pings +5 Pongs) giving an average transmission delay of 1.3 seconds between hosts.

We ran the experiment again at the beginning of Jan. 2002 and obtained the following results (Table 4) after letting the servent warm up for about 5 minutes. The average number of responding hosts was 91 . This time, the average delay per message transmission is $1.75 \mathrm{sec}$. 
Table 4. Hop Count and Delay in Pong Messages

\begin{tabular}{lccc}
\hline & Average & Minimum & Maximum \\
\hline Hop Count & 5.5 & 1 & 8 \\
Delay (sec.) & 19.1 & 0.8 & 238 \\
\hline
\end{tabular}

\section{Measuring the Performance of Gnutella Applications}

Beyond simply understanding the factors affecting the Gnutella network, our research is also aimed at improving the performance of applications. However, as demonstrated in our previous experiments, the performance of any one session depends on chance and measures of performance can therefore vary widely. Furthermore, the activity on the network varies with time. To be able to evaluate the effect of various servent parameters or strategies, we had to develop a methodology that would mitigate these problems. We wanted a methodology that would:

1. not disrupt the normal operations of the network,

2. reduce the effect of randomness on performance indicators, and

3 . enable the comparison of different versions of a servent.

The first requirement is partly resolved by the use of Jtella. This is not sufficient, however; when modifying the behavior of the Jtella servent, we had to be careful to act as a good netizen and supply all the information required by other servents. In the case of rejected connections in particular, we provided a list of addresses to the servent requesting the connection.

Our experiments described so far have clearly demonstrated that servent performance varies greatly over time. Running the servent over long periods, running multiple experiments at different times of the day and on different days of the week, and using averages from these runs overcomes in part the stochastic behavior of Gnutella.

The third requirement is the most difficult to fulfil. Clearly, we cannot compare two executions done at different time of the day or on different days, since there is no guaranty that the Gnutella network will be in the same state. Our solution is to run test programs in parallel with a fixed benchmark and to consider the relative performance.

Another basic problem is choice of a measure of performance. Over the course of our study, we used several indicators:

- the total number of messages,

- the total number of Pings,

- the total number of Pongs,

- the average horizon, and

- the number of distinct host addresses found.

No measure stood out as a best indicator. As a result we used them all and gave them equal weight. This yields the following experimental methodology:

- for each parameter value (or strategy) that we wish to test, we run an experiment,

- an experiment lasts 24 hours, and consists of 24 runs (of 45 minutes), once every hour, 
- for each run, we launch two (2) servents in parallel, a benchmark servent and a test servent,

- the parameters of the benchmark servent are constant for all experimental runs and serves as a basis for comparison,

- on each run, for each servent, we record the values of the 5 indicators listed above,

- the statistics collected serve to compute the performance ratio, noted $r$, of the test servent:

$$
r=\frac{1}{m} \cdot \sum_{i=1}^{m} \frac{\sum_{j=1}^{24} x_{i j}^{t}}{\sum_{j=1}^{24} x_{i j}^{b}}
$$

where

- $m$ is the number of indicators used,

- $x_{i j}^{s}$ is the value of indicator $i$ collected at the $j^{\text {th }}$ run of servent $s$, where $s$ equals $b$ for the benchmark servent and $t$ for the test servent.

We conducted a preliminary evaluation of this methodology to assess how the targeted number of connections $(K)$ influenced the performance of a servent. We expected that performance should increase with $K$ and perhaps taper off for very large values as bandwidth limitations start to play a role. We ran a series of experiments where the test servent used different values for the number of open connections. In particular, we had $K \in\{3,4,6,8,10,15\}$ while the benchmark servant used a fixed value of five open connections $(K=5)$.

The results of the experiment are quite compelling and appear to be linear (Fig. 5). A linear regression analysis on these results yields the following relation between $r$ and $K$ :

$$
r=0.2763 \cdot(K-1.680)
$$

From this experiment, we observe that a servent with a target of 2 connections or less would not receive any traffic $\left(k_{0}=1.680\right)$. It seems that 2 connections are always occupied trying to establish connections over the Gnutella networks. We also see that the use of more open connections increases in $r$.

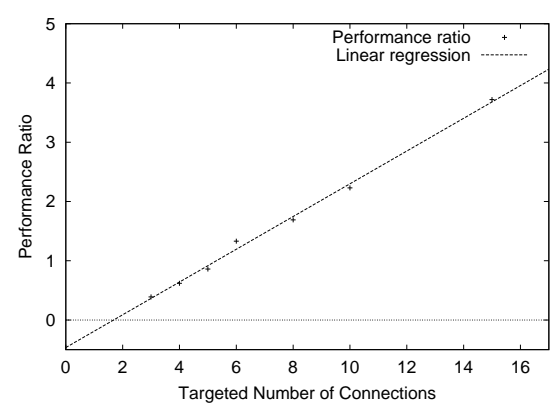

Fig. 5. Performance Ratio $(r)$ vs. Targeted Number of Connections $(K)$ 


\section{Conclusion}

We have investigated the behaviour of participants in Gnutella, a well-known Internet community. Although some of the phenomena observed are particular to Gnutella, many of our results are relevant to other communities.

An important observation is the highly random nature of network behaviour. Repeatedly we observed a "whales and minnows" phenomenon whereby average measurements are determined by a small number of rare events with huge values and are therefore neither representative of the rare events nor of the more common small values. For example, we measured the average duration of a session to be 31 seconds, but $1 \%$ of the sessions average $3000 \mathrm{sec}$. whereas the majority (99\%) average $1.3 \mathrm{sec}$. It is thus very difficult to get reproducible results.

Our experiments also showed that the composition of the community changes quite rapidly. Contrary to published results that suggest that connections last in the order of minutes or even hours [21], we found that sessions are much shorter: the duration of the average (median) session is less than half a second: $0.17 \mathrm{sec}$ in one case and $0.4 \mathrm{sec}$ in the other; and in another experiment we found that $98.7 \%$ of the sessions lasted less than 2 seconds.

To maintain connectivity, nodes continuously exchange addresses of connected hosts that can be used to replace failed neighbours. A surprising observation was that a large proportion of the information thus obtained is incorrect or redundant: 80 to $90 \%$ in the case of Gnutella's Pongs. A major part of the problem comes from hosts on subnetworks sending local (NAT) addresses which have no global validity. Even after filtering out flawed addresses, only a third of connection attempts result in a valid connection.

More generally, collaborative behaviour requires the exchange of organizational data between participants but flawed information may be a fact of life in open systems with unscreened participants, evolving technology, and a wide variety of software implementations.

Mapping the network or even estimating a horizon (the reachable portion of the net) may be more difficult than is generally believed. $2 / 3$ of our "good" hosts never acknowledged a Ping; other nodes do not forward Pings to their neighbours but return addresses from a local cache. The maximum horizon that we measured during our tests was less than 1000 nodes for 1 minute peaks. Average horizon values were much lower, normally in the hundreds.

We discovered semi-permanent reliable hosts but again they are rare. Starting from 42,000 site addresses we ended up with only 57 sites that were up $50 \%$ of the time or better.

We developped an effective methodology - based on comparative measures and replication - to overcome the stochastic nature of network activity and allow the evaluation of various operating strategies.

In conclusion, the experimental investigation of Gnutella has revealed many interesting technical findings as well as conceptual insights. It became clear that a local intelligent screening and processing of community information is central for efficiency as well as scalability of such networks. Future work will thus concentrate on evaluat- 
ing more sophisticated policies and strategies in both the real world of Gnutella and in simulated environments.

\section{Acknowledgements}

We would like to thank Cirano (Center for Inter-university Research and Analysis of Organizations, Montreal) where J. Vaucher is on sabbatical leave, for providing the machines and environment for this research.

Matthias Klonowski, an exchange student from Rostock, Germany, worked on the Jtella software during the summer of 2001. He pointed out several performance bottlenecks and helped discover a critical Java Bug.

\section{References}

1. The gnutella protocol specification v0.4. Clip2 Distributed Search Services. Available from http://www9.limewire.com/developer/gnutella_protocol_0.4.pdf.

2. What is gnutella. LimeWire LLC, 2001. Available from http://www.limewire.com/index.jsp/what_gnut.

3. Gnutella network size (realtime graph). LimeWire LLC, 2002. Available from http://www.limewire.com/.

4. E. Bonabeau, G. Theraulaz, J. Deneubourg, S. Aron, and S. Camazine. Selforganization in social insects. Trends in Ecol. Evol. 188-193, 1997.

5. I. Clarke, O. Sandberg, B. Wiley, and T. Hong. Freenet: a distributed anonymous information storage and retrieval system. In ICSI Workshop on Design Issues in Anonymity and Unobservability, Berkeley, CA, 2000.

6. Gary Flake, Steve Lawrence, and C. Lee Giles. Efficient identification of web communities. In Sixth ACM SIGKDD International Conference on Knowledge Discovery and Data Mining, pages 150-160, Boston, MA, August 20-23 2000.

7. L. N. Foner. YENTA: A multi-agent referral system for matchmaking. In First International Conference on Autonomous Agents, Marina del Rey, California, 1997.

8. David Gibson, Jon M. Kleinberg, and Prabhakar Raghavan. Inferring web communities from link topology. In UK Conference on Hypertext, pages 225-234, 1998.

9. Francis Heylighen. Collective intelligence and its implementation on the web: Algorithms to develop a collective mental map. Computational \& Mathematical Organization Theory, 5(3):253-280, 1999.

10. S. Ravi Kumar, Prabhakar Raghavan, Sridhar Rajagopalan, and Andrew Tomkins. Trawling the web for emerging cyber-communities. WWW8 / Computer Networks, 31(11-16):14811493, 1999.

11. K. McCrary. The gnutella file-sharing network and java. JavaWorld, 2000. Available from http://www.javaworld.com/javaworld/jw-10-2000/jw-1006-fileshare.html.

12. K. McCrary. Jtella: a java api for gnutella, 2000. Available from http://www.kenmccrary.com/jtella/index.html.

13. V. Menko, D. J. Neu, and Q. Shi. AntWorld: a collaborative web search tool. In Kropf et al., editor, Distributed Communities on the Web (DCW). Springer Verlag Berlin, 2000.

14. Stanley Milgram. The small-world problem. Psychology Today, 1967.

15. S. Osokine. Gnutella blown away? not exactly, 2001. Available from http://www.openp2p.com/pub/a/p2p/2001/07/11/numbers.html. 
16. Y. Rekhter, B. Moskowitz, D. Karrenberg, G. J. de Groot, and E. Lear. Address allocation for private internets. Request for Comments 1918, Internet Engineering Task Force, February 1996. Available from ftp://ftp.isi.edu/in-notes/rfc1918.txt.

17. M. Ripeanu. Peer-to-peer architecture case study: Gnutella network. Computer Science Dept., University of Chicago, 2001. Available from http://www.cs.uchicago.edu/files/tr_authentic/TR-2001-26.pdf.

18. J. Ritter. Why gnutella can't scale. no, really, 2001. Available from http://www.darkridge.com/jpr5/doc/gnutella.html.

19. C. Rohrs. Limewire design. LimeWire LLC, 2001. Available from http://www.limewire.org/project/www/design.html.

20. C. Rohrs and V. Falco. Ping/pong scheme. Technical paper, LimeWire LLC, 2001. Available from http://www.limewire.com/index.jsp/pingpong.

21. S. Saroiu, P. Gummadi, and S. Gribble. A measurement study of peer-to-peer file sharing systems. Technical report UW-CSE-01-06-02, University of Washington, 2002.

22. J. Sullivan. Napster: Music is for sharing. Wired News, November 1999. Available from http://www.wired.com/news/print/0,1294,32151,00.html.

23. J. Vaucher and M. Klonowski. Bug \#4508232: Unborn threads are not garbage collected. Sun Developper Connection, 2001. Available from http://developer.java.sun.com/developer/bugParade/bugs/4508232.html. 


\section{Liste des publications au CIRANO*}

Série Scientifique / Scientific Series (ISSN 1198-8177)

2002s-55 Experimenting with Gnutella CommunitiesJean Vaucher, Gilbert Babin, Peter Kropf, Thierry Jouve

2002s-54 Incentives? The Effect of Profit Sharing Plans Offered by Previous Employers on Current Wages / Daniel Parent

2002s-53 Multi-Task Learning For Option Pricing / J. Ghosn et Y. Bengio

2002s-52 Input Decay: Simple and Effective Soft Variable Selection / N. Chapados et Y.

Bengio

2002s-51 On Out-of-Sample Statistics for Time-Series / F. Gingras, Y. Bengio et C. Nadeau

2002s-50 Forecasting Non-Stationary Volatility with Hyper-Parameters / Y. Bengio et C. Dugas

2002s-49 Cost Functions and Model Combination for VaR-based Asset Allocation using Neural Networks / N. Chapados et Y. Bengio

2002s-48 Experiments on the Application of IOHMMs to Model Financial Returns Series / Y. Bengio, V.-P. Lauzon et R. Ducharme

2002s-47 Valorisation d'Options par Optimisation du Sharpe Ratio / Y. Bengio, R. Ducharme, O. Bardou et N. Chapados

2002s-46 Incorporating Second-Order Functional Knowledge for Better Option Pricing / C. Dugas, Y. Bengio, F. Bélisle, C. Nadeau et R. Garcia

2002s-45 Étude du Biais dans le Prix des Options / C. Dugas et Y. Bengio

2002s-44 Régularisation du Prix des Options : Stacking / O. Bardou et Y. Bengio

2002s-43 Monotonicity and Bounds for Cost Shares under the Path Serial Rule / Michel Truchon et Cyril Téjédo

2002s-42 Maximal Decompositions of Cost Games into Specific and Joint Costs / Michel Moreaux et Michel Truchon

2002s-41 Maximum Likelihood and the Bootstrap for Nonlinear Dynamic Models / Sílvia Gonçalves, Halbert White

2002s-40 Selective Penalization Of Polluters: An Inf-Convolution Approach / Ngo Van Long et Antoine Soubeyran

2002s-39 On the Mediational Role of Feelings of Self-Determination in the Workplace: Further Evidence and Generalization / Marc R. Blais et Nathalie M. Brière

2002s-38 The Interaction Between Global Task Motivation and the Motivational Function of Events on Self-Regulation: Is Sauce for the Goose, Sauce for the Gander? / Marc R. Blais et Ursula Hess

2002s-37 Static Versus Dynamic Structural Models of Depression: The Case of the CES-D / Andrea S. Riddle, Marc R. Blais et Ursula Hess

* Consultez la liste complète des publications du CIRANO et les publications elles-mêmes sur notre site Internet : 
2002s-36 A Multi-Group Investigation of the CES-D's Measurement Structure Across Adolescents, Young Adults and Middle-Aged Adults / Andrea S. Riddle, Marc R. Blais et Ursula Hess

2002s-35 Comparative Advantage, Learning, and Sectoral Wage Determination / Robert Gibbons, Lawrence F. Katz, Thomas Lemieux et Daniel Parent

2002s-34 European Economic Integration and the Labour Compact, 1850-1913 / Michael Huberman et Wayne Lewchuk

2002s-33 Which Volatility Model for Option Valuation? / Peter Christoffersen et Kris Jacobs

2002s-32 Production Technology, Information Technology, and Vertical Integration under Asymmetric Information / Gamal Atallah

2002s-31 Dynamique Motivationnelle de l'Épuisement et du Bien-être chez des Enseignants Africains / Manon Levesque, Marc R. Blais, Ursula Hess

2002s-30 Motivation, Comportements Organisationnels Discrétionnaires et Bien-être en Milieu Africain : Quand le Devoir Oblige / Manon Levesque, Marc R. Blais et Ursula Hess

2002s-29 Tax Incentives and Fertility in Canada: Permanent vs. Transitory Effects / Daniel Parent et Ling Wang

2002s-28 The Causal Effect of High School Employment on Educational Attainment in Canada / Daniel Parent

2002s-27 Employer-Supported Training in Canada and Its Impact on Mobility and Wages / Daniel Parent

2002s-26 Restructuring and Economic Performance: The Experience of the Tunisian Economy / Sofiane Ghali and Pierre Mohnen

2002s-25 What Type of Enterprise Forges Close Links With Universities and Government Labs? Evidence From CIS 2 / Pierre Mohnen et Cathy Hoareau

2002s-24 Environmental Performance of Canadian Pulp and Paper Plants : Why Some Do Well and Others Do Not ? / Julie Doonan, Paul Lanoie et Benoit Laplante

2002s-23 A Rule-driven Approach for Defining the Behavior of Negotiating Software Agents / Morad Benyoucef, Hakim Alj, Kim Levy et Rudolf K. Keller

2002s-22 Occupational Gender Segregation and Women's Wages in Canada: An Historical Perspective / Nicole M. Fortin et Michael Huberman

2002s-21 Information Content of Volatility Forecasts at Medium-term Horizons / John W. Galbraith et Turgut Kisinbay

2002s-20 Earnings Dispersion, Risk Aversion and Education / Christian Belzil et Jörgen Hansen

2002s-19 Unobserved Ability and the Return to Schooling / Christian Belzil et Jörgen Hansen

2002s-18 Auditing Policies and Information Systems in Principal-Agent Analysis / MarieCécile Fagart et Bernard Sinclair-Desgagné

2002s-17 The Choice of Instruments for Environmental Policy: Liability or Regulation? / Marcel Boyer, Donatella Porrini

2002s-16 Asymmetric Information and Product Differentiation / Marcel Boyer, Philippe Mahenc et Michel Moreaux

2002s-15 Entry Preventing Locations Under Incomplete Information / Marcel Boyer, Philippe Mahenc et Michel Moreaux 